Pure and Applied Mathematics Quarterly

Volume 7, Number 4

(Special Issue:

In memory of Eckart Viehweg)

$1085-1103,2011$

\title{
Symmetric Differentials of Rank 1 and Holomorphic Maps
}

\author{
Fedor Bogomolov* and Bruno De Oliveira** \\ To the memory of our esteemed colleague Eckart Viehweg
}

\begin{abstract}
The goal of this work is to develop the Albanese theory for symmetric differentials $w$ of rank 1 (i.e. locally $\left.w\right|_{U}=f \mu^{m}$, where $\mu \in H^{0}\left(U, \Omega_{X}^{1}\right)$ and $\left.f \in \mathcal{O}(U)\right)$. To accomplish this, the notion of closed symmetric differential is introduced and symmetric differentials of rank 1 are proved to be closed. It is then shown that for each differential of rank $1 w$ on a projective variety $X$ there is a holomorphic map $a_{w}: X \rightarrow A_{w} / \mathbb{Z}_{d}$ from $X$ to a quotient of an abelian variety $A_{w}$ by a cyclic group $\mathbb{Z}_{d}, d \mid m$, with isolated singularities such that $w=a_{w}{ }^{*}(u)$ and $u \in H^{0}\left(A_{w} / \mathbb{Z}_{d}, S_{\mathrm{orb}}^{m} \Omega_{A_{w} / \mathbb{Z}_{d}}^{1}\right)$. It is also shown that the presence of a differential of rank 1 implies the existence of a divisor $E \subset X$ with negative properties such that $\pi_{1}(X \backslash E)$ is an infinite group.
\end{abstract}

Keywords: Symmetric differentials, Albanese, closed differentials.

Received: Nov. 12, 2010; Revised: Jan. 17, 2011.

* Partially supported by the NSF grant DMS-1001662. ** Partially supported by the NSF grant DMS-0707097. The first author also acknowledges the support of AG Laboratory HSE grant RF goverment ag.1111.g34.31.0023 and the second author also thanks the financial support of Harvard University and Professor Yum-Tong Siu for the Spring of 2009. 


\section{INTRODUCTION}

Let $X$ be a projective manifold of dimension $n$, a symmetric differential of degree $m$ is a section of the m-th symmetric power of the sheaf of holomorphic 1-forms, $S^{m} \Omega_{X}^{1}$. A symmetric differential $w$ of degree $m$ is of rank 1 if it can be locally written in the form $\left.w\right|_{U}=f \mu^{m}$, where $\mu \in H^{0}\left(U, \Omega_{X}^{1}\right)$ and $f \in \mathcal{O}(U)$. Symmetric differentials of degree 1, i.e. holomorphic 1-forms, are trivially of rank 1. Holomorphic 1-forms $\mu$ on compact projective manifolds $X$ have the following properties:

i) $\mu \in H^{0}\left(X, \Omega_{X}^{1}\right)$ are closed (and locally exact).

ii) The presence of a nontrivial holomorphic 1-form $\mu$ implies the existence of a holomorphic map to an abelian variety $A(X), f: X \rightarrow A(X)$ and $\mu=f^{*} u$ with $u \in H^{0}\left(A(X), \Omega_{A(X)}^{1}\right)$.

iii) The presence of a nontrivial holomorphic 1-form $\mu$ imply that the abelianization of $\pi_{1}(X)$ is infinite.

In this work we will give an extension of these properties for rank 1 symmetric differentials of all degrees.

The notions of closed and locally exact symmetric differentials will be generalized to symmetric differentials of all degrees (and arbitrary rank). In essence a symmetric differential $w$ of degree $m$ is closed (locally exact) if around the general point (all points) of $X w$ is the product of $m$ exact 1-differentials. It will be shown that any symmetric differential of rank $1, w$, on a projective manifold $X$ is locally of the form $\left.w\right|_{U}=(d f)^{\otimes m}$, outside of a locus of codimension 1 . Hence $w$ is closed, but not necessarily locally exact. This result will be derived from the existence of a ramified covering $g: X^{\prime} \rightarrow X$ for which $g^{*} w=\mu^{m}$, where $\mu$ is a holomorphic 1-form on $X^{\prime}$. As a consequence, one has that the singular foliation $\mathcal{F}_{w}$ defined by $w$ either has a nonalgebraic integral or gives a fibration over a curve.

With respect to property ii) it will be shown that the presence of a nontrivial symmetric differential, $w$, of rank 1 of degree $m$ on a projective manifold $X$ implies the existence a holomorphic map into a quotient of an abelian variety $A_{w}$ by a cyclic group, $f: X \rightarrow A_{w} / \mathbb{Z}_{d}(d \mid m)$, and $w$ is is the pullback of an orbifold symmetric differential on $A_{w} / \mathbb{Z}_{d}$.

The topological property iii) will have the following counterpart: there is a divisor $E$ which is contracted by the map $f: X \rightarrow A_{w} / \mathbb{Z}_{d}$ for which $\pi_{1}(X \backslash$ $E$ ) is infinite (in fact, $\pi_{1}(X \backslash E)$ has a subgroup of finite index with infinite abelianization). The divisor $E$ can be chosen to have the following negative property: if $S$ is a general complete intersection of $X$ of dimension 2, then $E \cap S$ 
is negative divisor of $S$. Thus if $X$ has dimension two, then there is a contraction of $X$ to compact singular complex surface $X^{\prime}$ with the image of $E$ consisting of a finite set of points. It is clear in the case when $\operatorname{dim} f(X)=2$ but we show that it holds even if $f(X)$ is a curve.

The above results are also extended to twisted symmetric differentials of rank 1, i.e. sections of $S^{m}\left(\Omega_{X}^{1}\right) \otimes L$, where $L \in P i c^{\tau}(X)$ is a $\mathbb{C}^{*}$-flat line bundle on $X$. Apart from their independent interest, twisted symmetric differentials of rank 1 appear in the decomposition of closed symmetric differentials of higher rank. Twisted symmetric differentials of rank 1 inherit some of the geometric properties of twisted holomorphic differentials which were studied by GreenLazarsfeld, Beauville and Simpson [GrLa87], [Be92], [Si93] to understand the cohomology locus $S^{1}(X)=\left\{L \in \operatorname{Pic}^{\tau}(X) \mid H^{1}(X, L) \neq 0\right\}$. The case where $L$ is torsion is similar to the nontwisted case. In the case where $L$ is nontorsion one obtains that the presence of a nontrivial $w \in H^{0}\left(X, S^{m} \Omega_{X}^{1} \otimes L\right)$ of rank 1 on $X$ implies the existence of a holomorphic map into a smooth curve of genus $\geq 1$, $p: X \rightarrow B$, such that $w$ is the pullback of twisted orbifold symmetric differential on the curve $B$. Moreover, there is a divisor $N$ with the same negative properties as $E$ above such that $\pi_{1}(X \backslash N)$ is hyperbolic and the induced foliation $\mathcal{F}_{w}$ gives a fibration over a curve.

\section{Closed symmetric differentials}

It is well known that in the case of holomorphic 1-forms, $\mu$, the presence of a nontrivial closed differential $\mu$ implies the topological property that the 1stBetti number $\operatorname{dim} H^{1}(X, \mathbb{C}) \neq 0$. The condition of $\mu$ being closed, is necessary since there are simply connected complex of manifolds of dimension $\geq 3$ with nontrivial holomorphic 1-forms. In the case $X$ is a compact kahler manifold, the connection between holomorphic 1-forms and the homology of $X$ is always present since all holomorphic 1-forms are closed and can be stated in the stronger form: the dimension of $H^{0}\left(X, \Omega_{X}^{1}\right)$ determines the 1st-Betti number of $X$.

For differentials of higher degree the kahlerian condition on $X$ is not sufficient to guarantee a link between the presence of symmetric differentials on $X$ and the topology of $X$. An example of this fact is the noninvariance of the space of symmetric differentials on families of smooth projective manifolds $X_{t}$ despite all $X_{t}$ having the same underlying topological manifold (see [BoDeO06] for an example of a family of simply connected projective surfaces for which the general member of the family has no symmetric differentials while some special members have infinitely many). 
To expect to derive topological properties one needs to introduce, as is done in the case of holomorphic 1-forms, the notion of closed symmetric differentials.

Definition 2.1. Let $w \in H^{0}\left(X, S^{m} \Omega_{X}^{1}\right)$ be a symmetric differential on a smooth complex manifold $X$. The symmetric differential $w$ is:

i) exact on an open subset $U$ if $\left.w\right|_{U}=\left(d f_{1}\right)^{m_{1}} \ldots\left(d f_{k}\right)^{m_{k}}$, where $\sum_{i=1}^{k} m_{i}=m$, $f_{i} \in \mathcal{O}(U)$ and $d f_{i} \wedge d f_{j} \neq 0$.

ii) locally exact on $X$ if there is an open covering of $X,\left\{U_{i}\right\}$, such that $\left.w\right|_{U_{i}}$ is exact.

iii) closed on $X$ if it is exact in a neighborhood of a general point of $X$.

\section{Remarks:}

i) the definition of a closed differential presented here is general (in particular, it is not specific to rank 1 symmetric differentials).

ii) the condition of a symmetric differential being closed or exact is not linear for degrees $\geq 2$.

iii) the condition of being locally exact and the condition of being closed coincide for symmetric differentials of degree 1 , but that is no longer true for higher degrees even in the case of rank 1 . This fact is reflected nontrivially on the topological geometric properties that can be derived from either type of symmetric differentials of rank 1 , see next section.

The next proposition describes the locus where a closed symmetric differential fails to be locally exact and describes the local form of closed but non locally exact symmetric differentials.

Proposition 2.2. Let $w \in H^{0}\left(X, S^{m} \Omega_{X}^{1}\right)$ be a closed symmetric differential of rank 1. The locus where $w$ is not locally exact is the divisor $D \subset(w)_{0}\left((w)_{0}=\right.$ $\sum_{i=1}^{k} m_{i} D_{i}$ the zero divisor $\left.w\right)$ with $D=\cup_{\alpha} D_{\alpha}, \alpha \in\left\{i \mid m \not m_{i}\right\}$.

Proof. Any $x \in X$ has a neighborhood $U_{x}$ where

$$
\left.w\right|_{U_{x}}=f \mu^{\prime m}
$$

with $f \in \mathcal{O}\left(U_{x}\right)$ and $\mu^{\prime} \in H^{0}\left(U_{x}, \Omega_{X}^{1}\right)$ not vanishing outside codimension 2. If $x \in X \backslash(w)_{0}$, then $f$ has an m-th root on $U_{x}$ (shrink $U_{x}$ if necessary) and one has

$$
\left.w\right|_{U_{x}}=\mu^{m}
$$


with $\mu \in H^{0}\left(X, \Omega_{X}^{1}\right)$. Since $w$ is closed, there is a neighborhood $U_{y}$ of a general point of $y \in U_{x}$ where $\left.w\right|_{U_{y}}=(d g)^{m}$. This implies that $\mu$ is closed on the whole $U_{x}$ and hence locally exact on $U_{x}$. The same argument also shows that if $x \in D_{0}$ and $f$ in (2.1) has locally an $m$-th root, then $w$ is exact on some open $U_{x}$ containing $x$. Hence by covering $X \backslash D$ with such $U_{x}$, it follows that $w$ is locally exact on $X \backslash D_{0}$.

If $x$ is a general point in $D$, then there is a sufficiently small neighborhood $U_{x}$ of $x$ and a local system of coordinates where $D \cap U_{x}=\left\{z_{1}=0\right\}$ and $f=z_{1}^{m_{\alpha}}$ with $m \bigwedge m_{\alpha}$. If $w$ was locally exact at $x$, then after possible shrinking the neighborhood $U_{x}$, the following holds $z_{1}^{m_{\alpha}} \mu^{\prime m}=(d h)^{m}$, where the left side is as in (2.1) and $h \in \mathcal{O}\left(U_{x}\right)$. This is impossible since the order of vanishing of the right side along $D$ is a multiple of $m$. Hence $D$ is the locus where $w$ is not locally closed.

The next step consists of showing that all symmetric differentials of rank 1 on projective manifolds are closed. This result will be a consequence of the result stating that there are ramified coverings where the pullback of the symmetric differentials of rank 1 are the $\mathrm{m}$-th power of a global holomorphic 1-forms. To achieve this last result it is useful to translate symmetric differentials of degree $m$ on $X$ to sections of the line bundle $\mathcal{O}_{\mathbb{P}\left(\Omega_{X}^{1}\right)}(m)$ on the $\mathbb{P}^{n-1}$-bundle $\mathbb{P}\left(\Omega_{X}^{1}\right)$ over $X$. Given a symmetric differential $w \in H^{0}\left(X, S^{m} \Omega_{X}^{1}\right)$ on $X$ we denote by:

$$
Z_{w} \subset \mathbb{P}\left(\Omega_{X}^{1}\right)
$$

the divisor of $\mathbb{P}\left(\Omega_{X}^{1}\right)$ defined by the zero locus of the section of $\mathcal{O}_{\mathbb{P}\left(\Omega_{X}^{1}\right)}(m)$ associated with $w$.

The subvariety $Z_{w}$ can be decomposed into the sum of the horizontal and vertical components relative to the projection $\pi: \mathbb{P}\left(\Omega_{X}^{1}\right) \rightarrow X$ :

$$
Z_{w}=Z_{w, h}+Z_{w, v}
$$

The vertical component is the pullback of the divisor of zeros of $w, Z_{w, v}=\pi^{*} D_{0}$. The differential $w$ is of rank 1 if the horizontal divisor is of the form:

$$
Z_{w, h}=m Z
$$

As described before (2.4) is equivalent to the symmetric differential $w$ to be locally of the form $\left.w\right|_{U}=f \mu^{m}$, with $\mu \in H^{0}\left(U, \Omega_{X}^{1}\right)$ and $f \in \mathcal{O}(U)$. A symmetric differential of rank 1 defines at every $x \in X \backslash D_{0}$ a single hyperplane in $T_{x} X$ passing through the origin (in general a symmetric differential of degree $m$ defines a cone on $T_{x} X$ defined by a homogeneous polynomial of degree $\mathrm{m}$ ). 
Theorem 2.3. Let $X$ be a projective manifold and $w \in H^{0}\left(X, S^{m} \Omega_{X}^{1}\right)$ symmetric differential of rank 1 , then the following holds:

i) if $m$ divides the multiplicity of all components of the zero divisor of $w$, then there is an unramified cyclic covering of degree $d \mid m, g: X^{\prime} \rightarrow X$, where $g^{*} w=\mu^{m}$ with $\mu \in H^{0}\left(X^{\prime}, \Omega_{X^{\prime}}^{1}\right)$.

ii) otherwise, there is a ramified covering of degree $d, d \mid m, g: X^{\prime} \rightarrow X$, where $X^{\prime}$ is smooth and $g^{*} w=\mu^{m}$ with $\mu \in H^{0}\left(X^{\prime}, \Omega_{X^{\prime}}^{1}\right)$. The covering $X^{\prime}$ can be such that it has a cyclic $\mathbb{Z}_{d}$ action and the quotient variety $X^{\prime} / \mathbb{Z}_{d}$ has a birational morphism onto $X$.

iii) $w$ is closed (moreover, it is locally exact in case i)).

Proof. Given a symmetric differential $w$ of degree $m$ and rank 1 on $X$, we can find a covering $\left\{U_{i}\right\}$ of $X$ where $\left.w\right|_{U_{i}}=f_{i} \mu_{i}^{\otimes m}, f_{i} \in \mathcal{O}\left(U_{i}\right)$ and $\mu_{i}=\Omega_{X}^{1}\left(U_{i}\right)$. If all the $f_{i}$ have an m-th root, then one has $\left.w\right|_{U_{i}}=\bar{\mu}_{i}^{\otimes m}$ with $\bar{\mu}_{i} \in \Omega_{X}^{1}\left(U_{i}\right)$. On the intersections $U_{i j}, \bar{\mu}_{i}=\epsilon_{i j} \bar{\mu}_{j}$, with $\epsilon_{i j}$ m-th roots of unity. Associated with the 1-cocycle $\left\{\epsilon_{i j}\right\} \in H^{1}\left(X, \mathbb{Z}_{m}\right)$ there is an unramified covering $g: X^{\prime} \rightarrow X$ of degree $d, d \mid m$, where the pullback of the 1-cocycle becomes cohomologous to zero. Hence one can multiply the pullbacks $g^{*} \bar{\mu}_{i}$ by m-th roots of unity to form a holomorphic 1-form $\mu$ on $X^{\prime}$ such that $\mu^{m}=g^{*} w$. Since global 1-forms on $X^{\prime}$ are closed, it follows that the $\bar{\mu}_{i}$ are closed and finally that $w$ is closed.

In the case some of the $f_{i}$ have no m-th in $\mathcal{O}\left(U_{i}\right)$, one needs to show that there is a smooth ramified covering $g: X^{\prime} \rightarrow X$ where the $f_{i}$ acquire m-th roots and $g^{*} w=\mu^{\otimes m}$ with $\mu \in H^{0}\left(X^{\prime}, \Omega_{X^{\prime}}^{1}\right)$.

The divisor $Z_{w}$ in $\mathbb{P}\left(\Omega_{X}^{1}\right)$ associated with $w$ has the decomposition $Z_{w}=$ $m Z+\pi^{*} D_{0}$, where $Z$ is an horizontal divisor and $D_{0}$ is the divisor of zeros of $w$. The line bundle $\mathcal{O}(Z)$ is of the form $\mathcal{O}(Z) \simeq \mathcal{O}_{\mathbb{P}\left(\Omega_{X}^{1}\right)}(1) \otimes \pi^{*} L$, where $L$ is a line bundle on $X$ satisfying $L^{m}=\mathcal{O}\left(-D_{0}\right)$, since $\mathcal{O}\left(Z_{w}\right) \simeq \mathcal{O}_{\mathbb{P}\left(\Omega_{X}^{1}\right)}(m)$. The twisted holomorphic 1-form $\tilde{\mu} \in H^{0}\left(X, \Omega_{X}^{1} \otimes L\right)$ corresponding to the canonical section of $\mathcal{O}(Z)$ satisfies $\tilde{\mu}^{m} \otimes s=w, s$ is the canonical section associated with the zero divisor $D_{0}$.

The desired covering $g: X^{\prime} \rightarrow X$ follows from the standard covering constructions (see [Bo78] and also [La01] for an overview of the covering constructions). The key step is building an intermediate covering $c: X^{\prime \prime} \rightarrow X$ for which $c^{*} s=\left(s^{\prime \prime}\right)^{m}$, where $s^{\prime \prime} \in H^{0}\left(X^{\prime \prime}, \mathcal{O}\left(D_{0}^{\prime \prime}\right)\right)$ and $m D_{0}^{\prime \prime}=c^{*} D_{0}$. Consider the irreducible decomposition of the zero divisor, $D_{0}=\sum_{i=1}^{k} m_{i} D_{i}$ and let $r=\operatorname{gcd}\left(m, m_{1}, \ldots, m_{k}\right)$. Then build the cyclic covering of $X$ associated with $\left(L^{*}\right)^{m / r}=\mathcal{O}\left(1 / r D_{0}\right)$, the reason for factoring $r$ is to make the cyclic covering irreducible but note that $X^{\prime \prime}$ is generically singular. The cyclic cover $c$ is such that $c^{*} L=\mathcal{O}\left(-D_{0}^{\prime \prime}\right)$ and $c^{*} D_{0}=m D_{0}^{\prime \prime}$. Let $s^{\prime \prime}$ be the canonical section of $\mathcal{O}\left(D_{0}^{\prime \prime}\right)$ such that $s^{\prime \prime m}=c^{*} s$. To finalize, let $g=\sigma \circ c: X^{\prime} \rightarrow X$ where $\sigma: X^{\prime} \rightarrow X^{\prime \prime}$ is a resolution of singularities of $X^{\prime \prime}$. Set $D_{0}^{\prime}=\sigma^{*} D_{0}^{\prime \prime}$ and $s^{\prime}=\sigma^{*} s^{\prime \prime}$ and $\mu=g^{*} \tilde{\mu} \otimes s^{\prime}$, 
then $\mu^{m}=g^{*} w$ as desired since $g^{*} L=\mathcal{O}\left(-D_{0}^{\prime}\right), m D_{0}^{\prime}=g^{*} D_{0}$ and $s^{\prime m}=g^{*} s$, completing step i).

The variety $X^{\prime}$ can be constructed with an action of $\mathbb{Z}_{d}$ since $X^{\prime \prime}$ has a cyclic action and one can do an equivariant of $X^{\prime \prime}$ ([AbWa97].

The claim iii) follows from i) and ii) since away from the ramification locus $w$ is locally the $\mathrm{m}$-th power of a holomorphic 1-form whose pullback by $g$ is closed.

A symmetric differential $w$ of rank 1 defines a foliation $\mathcal{F}_{w}$ whose leaves are the hypersurfaces on which the pullback of $w$ vanishes. Using theorem 2.3 one has that there is a ramifified cover for which the pre-image of the leaves of $\mathcal{F}_{w}$ are the leaves of a foliation defined by a holomorphic 1-form, this leads to:

Corollary 2.4. A symmetric differential of rank $1, w \in H^{0}\left(X, S^{m} \Omega_{X}^{1}\right)$, defines a singular holomorphic foliation $\mathcal{F}_{w}$ on $X$ with the property that either:

i) $\mathcal{F}_{w}$ has a nonalgebraic integral, or

ii) $\mathcal{F}_{w}$ gives a fibration over a curve.

Proof. Theorem 2.3 gives a cyclic ramified covering $f: X^{\prime} \rightarrow X$ for which $f^{*} w=$ $\mu^{\otimes m}$ with $\mu \in H^{0}\left(X^{\prime}, \Omega_{X^{\prime}}^{1}\right)$. The pre-image of the leaves of the foliation $\mathcal{F}_{w}$ are the leaves of the foliation $\mathcal{F}_{\mu}$ defined by the holomorphic 1-form $\mu$ on $X^{\prime}$, for which the desired dichotomy holds. If $\mathcal{F}_{w}$ has a 1-dimensional family of compact leaves, then so does $\mathcal{F}_{\mu}$ and hence there is a map onto a curve $h: X^{\prime} \rightarrow C^{\prime}$ whose fibers are the leaves of $\mathcal{F}_{\mu}$. Moreover the map $h$ comes from the Albanese map $a_{X^{\prime}}: X^{\prime} \rightarrow A\left(X^{\prime}\right)$ composed with the quotient map $p: A\left(X^{\prime}\right) \rightarrow A / T_{\mu}$, where $T_{\mu}$ is the maximal abelian subvariety of $A\left(X^{\prime}\right)$ contained in a leaf of the form $\mu^{\prime} \in H^{0}\left(A\left(X^{\prime}\right), \Omega_{A\left(X^{\prime}\right)}^{1}\right)$ whose pullback is $a_{X^{\prime}}^{*} \mu^{\prime}=\mu$. The cyclic action on $X^{\prime}$ induces a cyclic action on $\mathrm{A}\left(\mathrm{X}^{\prime}\right)$ which descends to $A / T_{\mu}$ ( $\mu^{\prime}$ is an eigenvector for the associated linear action on $\left.H^{0}\left(A\left(X^{\prime}\right), \Omega_{A\left(X^{\prime}\right)}^{1}\right)\right)$. The curve $C^{\prime}$ is just the image of $X^{\prime}$ under the map $p \circ a_{X^{\prime}}$ which is an equivariant map for the cyclic action, hence the map $h: X^{\prime} \rightarrow C^{\prime}$ descends to a map $f: X \rightarrow C$, where $C$ is the cyclic quotient of $C^{\prime}$, giving the desired fibration on $X$.

\section{HOLOMORPHIC MAPS AND TOPOLOGICAL PROPERTIES}


The purpose of this section is to describe the complex geometric and topological properties associated with the presence of symmetric differentials of rank 1 on a projective manifold.

\subsection{Structure theorem.}

As a starting point, it will be shown that if a complex manifold $X$ has a symmetric differential of rank 1 that is locally exact, then there are purely topological implications on $X$.

Proposition 3.1. Let $X$ be a compact complex manifold with a nontrivial locally exact $w \in H^{0}\left(X, S^{m} \Omega_{X}^{1}\right)$ of rank 1. Then $\pi_{1}(X)$ is infinite, in fact, $\pi_{1}(X)$ has a subgroup with infinite abelianization.

Proof. The condition of local exactness on $w$ states that it exists an open covering of $X\left\{U_{i}\right\}$ where $\left.w\right|_{U_{i}}=\left(d f_{i}\right)^{m}$. Hence

$$
d f_{i}=\epsilon_{i j} d f_{j}
$$

on $U_{i j}$ with $\epsilon_{i j}$ an m-th root of unity. Associated with the 1-cocycle $\left\{\epsilon_{i j}\right\}$ in $H^{1}\left(X, \mathbb{Z}_{m}\right)$, there is a flat line bundle $L_{\epsilon}$ and a finite unramified d-covering with $d \mid m, f: X^{\prime} \rightarrow X$, where the pullback $f^{*} L_{\epsilon} \simeq \mathcal{O}_{X^{\prime}}$. The condition (3.1) gives a nontrivial element in $\mu \in H^{0}\left(X, \Omega_{X}^{1} \otimes L_{\epsilon}\right)$ and hence a nontrivial element of $f^{*} \mu \in H^{0}\left(X^{\prime}, \Omega_{X^{\prime}}^{1}\right)$ since $f^{*} L_{\epsilon} \simeq \mathcal{O}_{X^{\prime}}$.

The conclusion follows from the fact that if the abelianization of $\pi_{1}\left(X^{\prime}\right)$ is finite, then the periods of $f^{*} \mu$ would be trivial and its integral would define a nonconstant holomorphic function on $X^{\prime}$.

It was shown in the previous section that the hypothesis of the above proposition hold if $X$ is a kahler manifold and $w$ is a m-symmetric differential of rank 1 for which all multiplicities of the components of its zero divisor are divisible by $m$.

As was described and announced in the previous section, there are global symmetric differentials of rank 1 which are not locally exact and different geometric properties should be expected from their presence. One of the key differences is that in contrast with the above proposition, there are simply connected compact kahler manifolds nontrivial symmetric differentials of rank 1.

Example: there are simply connected surfaces with nontrivial closed differentials. The example to be described is inspired in our previously example [BDeO06]. Let $A^{3}$ be a 3 -dimensional abelian variety. Consider a surface $X$ which is a smooth 
hypersurface section of $A^{3}$ of sufficiently high degree, invariant under the natural involution $\theta=-i d$ and passes through one and only one (for simplicity) of the fixed points of $\theta$, call this point $p$. The claim is that the minimal resolution of $\tilde{X}$ of $X / \theta$ is one of the desired examples.

The manifold $\tilde{X}$ is simply connected since $H^{0}\left(\tilde{X}, \Omega_{\tilde{X}}^{1}\right)=H^{0}\left(X, \Omega_{X}^{1}\right)^{\theta}=0$ (there are no invariant forms under $\theta$ ) and $\pi_{1}(\tilde{X})$ is abelian. The fundamental group $\pi_{1}(\tilde{X})$ is abelian since $\pi_{1}(X)=\pi_{1}\left(A^{3}\right)=H_{1}\left(A^{3}, \mathbb{Z}\right), \pi_{1}(X) \rightarrow \pi_{1}(X / \theta)$ is surjective (every loop with base point the singular point of $X / \theta$ lifts to a loop with base point the fixed point of $X$ by $\theta)$ and $\pi_{1}(\tilde{X})=\pi_{1}(X / \theta)$ (the exceptional locus is $\left.\mathbb{P}^{1}\right)$.

To produce a nontrivial symmetric differential of rank 1 on $\tilde{X}$ one picks the 1differential $w \in H^{0}\left(A^{3}, \Omega_{A^{3}}^{1}\right)$ which is trivial on $T_{p} X$, then $w^{2}$ induces the desired differential on $\tilde{X}$. Denote the induced differential on $X$ induced by $w$ also by $w$. The differential $w^{2}$ is invariant under the involution and hence it induces a differential $\tilde{w}^{2}$ on $\tilde{X} \backslash E$, where $E$ is the $(-2)$-curve over the nodal singularity of $X / \theta$. What remains to be shown is that $\tilde{w}^{2}$ extends to a holomorphic differential of degree 2 on the whole $\tilde{X}$.

To see this we opt for an explicit description. After the appropriate choice of coordinates around $p$ on $A^{3}, X$ can be locally described by $\left\{z_{3}=f\left(z_{1}, z_{2}\right)\right\}$ where:

$$
f\left(z_{1}, z_{2}\right)=\sum_{l=1}^{\infty} \sum_{m_{1}+m_{2}=2 l+1} a_{m_{1} m_{2}} z_{1}^{m_{1}} z_{2}^{m_{2}}
$$

where $l \geq 1$ (this form guarantees invariance under $\theta$ and $\left.d z_{3}\right|_{T_{x} X}=0$ ). The differential $w$ is given by $w=d z_{3}=d f$. Let $X^{\prime}$ be the auxiliary surface obtained by blowing up $X$ at $p$. This surface comes with the blow up map at $p, \sigma: X^{\prime} \rightarrow$ $X$, and a double cover map onto $\tilde{X}, g: X^{\prime} \rightarrow \tilde{X}$. A point in $\sigma^{-1}(p)$ has a neighborhood $(U,(u, v))$ on which $\sigma(u, v)=(u, u v)$ and $g(u, v)=\left(u^{2}, v\right)$. The condition for an invariant symmetric differential on $X^{\prime}$ to be the pullback of a holomorphic differential on $\tilde{X}$ is that the coefficients of the terms $(d u)^{k}(d v)^{m-k}$ must vanish along $u=0$ with multiplicity at least $\mathrm{k}$. This is guaranteed for $\sigma^{*} w^{2}$ since $\sigma^{*} f=u^{r} h(u, v)$ with $r \geq 3$ by (3.2). Note that since the Taylor expansion of $\sigma^{*} f$ have always $u$ to an odd power, that the differential $\tilde{w}^{2}$ on $\tilde{X}$ induced from $\left(\sigma^{*} d f\right)^{2}$ can not be locally exact along $E$.

The example just described has the key characteristics of the general case as the following result shows. 
Theorem 3.2. (Structure Theorem) Let $X$ be a projective manifold and $w \in$ $H^{0}\left(X, S^{m} \Omega_{X}^{1}\right)$ a nontrivial symmetric differential of rank 1. If $(w)_{0}=\sum_{i} l_{i} D_{i}$, $D_{i}$ irreducible, is the zero divisor of $w$ and $E=\sum_{j \in\left\{i \mid m \nmid l_{i}\right\}} D_{j}$, then:

i) There is a holomorphic map from $X$ to a quotient with isolated singularities of an abelian variety $A_{w}$ by a cyclic group $\mathbb{Z}_{d}$ with $d \mid m, a_{w}: X \rightarrow A_{w} / \mathbb{Z}_{d}$, such that $w=a_{w}{ }^{*}(u)$ and $u \in H^{0}\left(A_{w} / \mathbb{Z}_{d}, S_{\mathrm{orb}}^{m} \Omega_{A_{w} / \mathbb{Z}_{d}}^{1}\right)$.

ii) $\pi_{1}(X \backslash E)$ is infinite. More precisely, $\pi_{1}(X \backslash E)$ has a normal subgroup $\Gamma$ for which $\pi_{1}(X \backslash E) / \Gamma$ is cyclic of order $\leq m$ and its abelianization, $\Gamma /[\Gamma, \Gamma]$, is an infinite group. The divisor $E$ is nonpositive in the sense that it is contracted to a point by the map $a_{w}: X \rightarrow A_{w} / \mathbb{Z}_{d}\left(\operatorname{dim} a_{w}(X) \geq 1\right)$.

Proof. Recall that theorem 2.3 constructed a covering $f: X^{\prime} \rightarrow X$ ramified over the divisor $E$ for which $f^{*} w=\mu^{m}, \mu \in H^{0}\left(X^{\prime}, \Omega_{X^{\prime}}^{1}\right)$. The degree $d$ of the constructed covering map $f$ is minimal among all the degrees of coverings $f: \tilde{X} \rightarrow X$ with the property that exists $\mu \in H^{0}\left(\tilde{X}, \Omega_{\tilde{X}}^{1}\right)$ satisfying $f^{*} w=\mu^{m}$. Moreover, the covering manifold $X^{\prime}$ is built with a $\mathbb{Z}_{d}$ action and the covering $f: X^{\prime} \rightarrow X$ factors through the quotient $X^{\prime} / \mathbb{Z}_{d}$ where the induced map $\sigma$ : $X^{\prime} / \mathbb{Z}_{d} \rightarrow X$ a birational morphism.

The universal property of the Albanese implies that the action of $\mathbb{Z}_{d}$ on $X^{\prime}$ induces an action on the Albanese variety of $X^{\prime}$ with respect to which the Albanese map $a_{X^{\prime}}: X^{\prime} \rightarrow A\left(X^{\prime}\right)$ is equivariant. The next two lemmas, of independent interest, will be used to build an invariant abelian subvariety $A_{\mu}$ of $A\left(X^{\prime}\right)$ with quotient $A=A\left(X^{\prime}\right) / A_{\mu}$ such that: a translate of each connected component of $E$ is contained in $A_{\mu} ; \mu$ is a pullback of a differential on $A ; A / \mathbb{Z}_{d}$ only has isolated singularities.

Lemma 3.3. Let $Y$ be a kahler manifold with a $\mathbb{Z}_{d}$ action, $a_{Y}: Y \rightarrow A(Y)$ the Albanese map and $u \in H^{0}\left(Y, \Omega_{Y}^{1}\right)$ an eigenvector associated with a faithful character of the induced action on $H^{0}\left(Y, \Omega_{Y}^{1}\right)$. Then there exists an invariant subtorus $i: T_{m} \hookrightarrow A(Y)$, such that $u$ is induced from $A(Y) / T_{m}$ and $\left[A(Y) / T_{m}\right] / \mathbb{Z}_{d}$ is an orbifold with isolated singularities.

Proof. The action of $\mathbb{Z}_{d}$ on $A(Y)$ is an affine action where the linear part of the action is the dual of the faithful, by the hypothesis, action of $\mathbb{Z}_{d}$ on $H^{0}\left(Y, \Omega_{Y}^{1}\right)$. Associated with the faithful linear action of $Z_{d}$ on $A(Y)$, one has a cyclic subgroup $<f>\subset \operatorname{End}(A(Y))$ isomorphic to $\mathbb{Z}_{d}$ where $f$ is the endomorphism associated with the action of a generator of $\mathbb{Z}_{d}$. From the action one also has the decomposition $H^{0}\left(Y, \Omega_{Y}^{1}\right)^{*}=\bigoplus_{i=0}^{d-1} E_{\chi_{i}}$, where the $\chi_{i}$ 's are the d characters of $\mathbb{Z}_{d}$.

Consider the endomorphisms $I-f^{s} \in \operatorname{End}(A(Y))$, where $s \mid d$ but $s \neq d$, the identity component of $\operatorname{Ker}\left(I-f^{s}\right)$ is a subtorus $T_{s} \subset A(Y)$. Let $T_{m}=\operatorname{Span}_{s} T_{s}$ be the subtorus consisting of the span of the subtori $T_{s}$ and $i: T_{m} \hookrightarrow A(Y)$ its 
embedding. Let $u^{\prime} \in H^{0}\left(A(Y), \Omega_{A(Y)}^{1}\right)$ be the differential such that $a_{Y}^{*} u^{\prime}=u$, then $i^{*} u^{\prime}=0$ since $T_{m}$ it is the subtorus associated with the linear subspace $\bigoplus_{j} E_{\chi_{j}}$ where the $\chi_{j}$ are not faithful characters. This implies that $u^{\prime}$ and hence $u$ are pullbacks of a differential $u^{\prime \prime}$ on the quotient torus $A(Y) / T_{m}$.

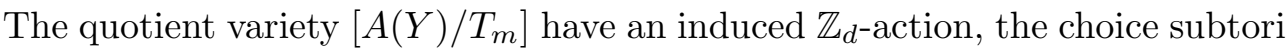
$T_{m}$ was to guarantee that all characters associated to this action are faithful. This implies that $\left[A(Y) / T_{m}\right] / \mathbb{Z}_{d}$ is an orbifold with only isolated singularities (the set of fixed points for the action is finite).

Lemma 3.4. Let $Y$ be a kahler manifold with an action of a finite group $G$, and $i: Z \hookrightarrow Y$ an invariant subvariety with connected components $\bar{Z}_{\alpha}$. Then there exists an invariant subtorus $T_{Z}$ of the Albanese $A(Y)$ with quotient $A(Y, Z)=$ $A(Y) / T_{Z}$ for which

$$
a_{Y, Z}^{*}\left(H^{0}\left(A(Y, Z), \Omega_{A(Y, Z)}^{1}\right)\right)=\left\{\mu \in H^{0}\left(Y, \Omega_{Y}^{1}\right) \mid \forall \alpha, \bar{Z}_{\alpha} \subset L_{\alpha}, L_{\alpha} \in \mathcal{L}_{\mu}\right\}
$$

where $a_{Y, Z}: Y \rightarrow A(Y, Z)$ is the composition of the Albanese map with the quotient map and $\mathcal{L}_{\mu}$ the set of leaves of $\mu$.

Proof. Let $a_{Y}: Y \rightarrow A(Y)$ be the Albanese map and let $Z=\cup Z_{i}, Z_{i}$ irreducible, $i_{i}: Z_{i} \hookrightarrow Y$ be the inclusion maps and $\sigma_{i}: \hat{Z}_{i} \rightarrow Z_{i}$ be resolutions of the $Z_{i}$. For each map $j_{i}=i_{i} \circ \sigma_{i}: \hat{Z}_{i} \rightarrow Y$ comes a Lie group homomorphism $a_{i}: A\left(\hat{Z}_{i}\right) \rightarrow A(Y)$. Each subtori $a_{i}\left(A\left(\hat{Z}_{i}\right)\right)$ is defined by the subspace $V_{i}$ of $H^{0}\left(A(Y), \Omega_{Y}^{1}\right)$ consisting of the differentials whose pullback to $Z_{i}$ is trivial. The subtorus $T_{Z}=\operatorname{Span}_{i}\left[a_{i}\left(A\left(\hat{Z}_{i}\right)\right)\right]$ by construction contains a translate of $a_{Y}\left(\bar{Z}_{\alpha}\right)$ of each connected component $\bar{Z}_{\alpha}$. Since the subvariety $Z$ is invariant this implies that the subspaces $V_{i}$ are permuted under the action and hence $T_{Z}$ is also invariant.

With respect to the equality (3.3), the inclusion $a_{Y, Z}^{*}\left(H^{0}\left(A(Y, Z), \Omega_{A(Y, Z)}^{1}\right)\right) \subset$ $\left\{\mu \in H^{0}\left(Y, \Omega_{Y}^{1}\right) \mid \forall \alpha, \bar{Z}_{\alpha} \subset L, L \in \mathcal{L}_{\mu}\right\}$ follows from the fact that a translate of $a_{Y}\left(\bar{Z}_{\alpha}\right)$ is contained in $T_{Z}$. To see the reverse inclusion, for each $\mu \in H^{0}\left(Y, \Omega_{Y}^{1}\right)$ denote by $\mu^{\prime} \in H^{0}\left(A(Y), \Omega_{A(Y)}^{1}\right)$ the differential for which $a_{Y}^{*} \mu^{\prime}=\mu$, the condition that each $\bar{Z}_{\alpha} \subset L$, with $L \in \mathcal{L}_{\mu}$ implies that $a_{i}\left(A\left(\hat{Z}_{i}\right)\right)$ are contained in leaves of $\mu^{\prime}$. Hence $T_{Z}$ is contained in a leaf of $\mu^{\prime}$ from which follows that $\mu^{\prime}$ is the pullback of a differential on $A(Y, Z)$ finishing the proof.

Returning to the proof of theorem we observe two facts:

1) the holomorphic 1-form $\mu$ satisfying $\mu^{\otimes m}=f^{*} w$ is an eigenvector associated with a faithful character of the $\mathbb{Z}_{d}$ action on $H^{0}\left(X^{\prime}, \Omega_{X^{\prime}}^{1}\right)$. This holds since $d$ is the minimal degree for the coverings where $f^{*} w$ has a m-th root. 
2) The pre-image of the ramification locus $f^{-1} E$ is $\mathbb{Z}_{d^{-}}$-invariant, in fact, the strict pre-image of $E$ is fixed by the action.

Let $A_{\mu^{\prime}}$ be the maximal abelian subvariety of $A\left(X^{\prime}\right)$ that is contained in a leaf of $\mu^{\prime}$, where $\mu^{\prime}$ is the differential on $A\left(X^{\prime}\right)$ such that $a_{X^{\prime}}^{*} \mu^{\prime}=\mu$. The subtorus $A_{\mu^{\prime}}$ is $\mathbb{Z}_{d^{-}}$invariant, since $\mu^{\prime}$ is an eigenvector for the action. Let $q: A\left(X^{\prime}\right) \rightarrow$ $A_{w}=A\left(X^{\prime}\right) / A_{\mu^{\prime}}$ be the standard quotient map and $a_{\mu}=q \circ a_{X^{\prime}}: X^{\prime} \rightarrow A_{w}$.

The abelian subvarieties $T_{m}$ and $T_{f^{-1}(E)}$ of $A\left(X^{\prime}\right)$ coming respectively from applying lemma 3.3 to pair $\left(X^{\prime}, \mu\right)$ and lemma 3.4 to the pair $\left(X^{\prime}, f^{-1}(E)\right)$ are contained in $A_{\mu^{\prime}}$ since they are both contained in the leaf of $\mu^{\prime}$ passing through the origin. Hence one has all the desired properties: there is $\mu^{\prime \prime} \in H^{0}\left(A_{w}, \Omega_{A_{w}}^{1}\right)$ such that $a_{\mu}{ }^{*} \mu^{\prime \prime}=\mu, a_{\mu}$ contracts each connected component of $f^{-1} E$ to a point and that the $\mathbb{Z}_{d}$ action descends to $A_{w}$ making $A_{w} / \mathbb{Z}_{d}$ an orbifold with only isolated singularities. Finally we have the diagram:

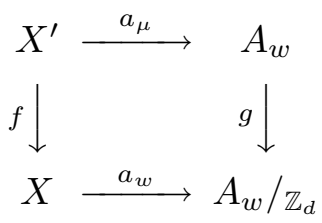

The $\mathbb{Z}_{d^{-}}$-invariant symmetric differential $\mu^{\prime \prime \otimes m}$ gives a section $u$ of the sheaf $S_{\text {orb }}^{m} \Omega_{A_{w} / \mathbb{Z}_{d}}^{1}$ of symmetric m-th differentials on the orbifold $A_{w} / \mathbb{Z}_{d}$ (by definition). The pullback of this symmetric differential is $w$ finishing the proof of i).

Part ii) is a direct consequence of the theorem 2.3 as the theorem implies that $H_{1}\left(X^{\prime}, \mathbb{Z}\right)$ is infinite and therefore $H_{1}\left(X^{\prime} \backslash f^{-1}(E), \mathbb{Z}\right)$ is also infinite. The induced covering $f: X^{\prime} \backslash f^{-1}(E) \rightarrow X \backslash E$ is a cyclic unramified covering of the degree $d$. Hence the exact sequence $0 \rightarrow \pi_{1}\left(X^{\prime} \backslash f^{-1}(E)\right) \rightarrow \pi_{1}(X \backslash E) \rightarrow \mathbb{Z}_{d} \rightarrow 0$ holds and part ii) follows. The part of $E$ being contracted follows from i).

Concerning the negative properties of the divisor $E$ appearing in the Structure Theorem one has two distinct cases: 1) $a_{w}\left(X^{\prime}\right)$ has dimension $\geq 2$, then the relevant negative property of the divisor $E$ is that $E$ is contracted via a holomomorphic map to a locus of codimension $\geq 2 ; 2) a_{w}\left(X^{\prime}\right)=1$, in this case $E$ needs not have negative properties. The next section addresses second case and it will be shown that one can find a divisor $N \subset E$ with negative properties, 


\subsection{Symmetric differentials induced from curves.}

We consider the case when the symmetric differential of rank $1, w$, on $X$ is induced from a curve $C$, i.e. there is a holomorphic map $f: X \rightarrow C$ and an orbifold symmetric differential $u$ in $C$ such that $f^{*} u=w$. This occurs for example when in the structure theorem the image $a_{w}(X) \subset A_{w} / \mathbb{Z}_{d}$ is curve. Note that in this case the foliation defined by $w$ is algebraic.

One of aims of this section is to show that also when $w$ is induced from a curve one can find a divisor $N$ on $X$ such that $\pi_{1}(X \backslash N)$ is infinite and $N$ has negative properties. Actually, the result shown will be stronger: in the case $w$ is induced from a curve one has: either $\pi_{1}(X)$ is infinite or exists an $N$ with the negative properties (a general complete intersection $S$ of dimension 2 in $X$ has $N \cap S$ as a negative divisor) such that $\pi_{1}(X \backslash N)$ is hyperbolic, i.e. it has a subgroup of finite index which surjects onto a free group $\mathbb{F}_{n}, n \geq 2$. The negative properties of $N$ follow from the fact that its connected components are properly contained in the fibers of $f$.

Theorem 3.2 for the case $w$ is induced from a curve gives in particular the following diagram:

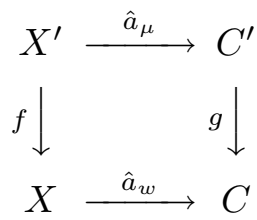

$C^{\prime}$ is the normalization of the curve $a_{\mu}\left(X^{\prime}\right)$ in $A_{w}$ and $C$ is the quotient of $C^{\prime}$ by $\mathbb{Z}_{d}$ (also smooth). The symmetric differential $w \in H^{0}\left(X, S^{m} \Omega_{X}^{1}\right)$ is the pullback $w=\hat{a}_{w} u$, where $u \in H^{0}\left(C, S_{\mathrm{orb}}^{m} \Omega_{C^{\prime} / \mathbb{Z}_{d}}^{1}\right)$.

To extract the desired geometric properties for $X$ (i.e. the existence of a divisor $N \subset X$ with negative properties such that $\pi_{1}(X \backslash N)$ is infinite) the only requirements needed on $X$ will be existence of a holomorphic map $f: X \rightarrow C$ such that the pullback of an orbifold symmetric differential on $C$ (relative to some orbifold structure on $C$ ) is holomorphic on $X$.

Theorem 3.5. Let $X$ be a projective manifold with a holomorphic map $f: X \rightarrow$ $C$ to a smooth curve $C$ such that there is an orbifold symmetric differential $u$ on $C$ with holomorphic pullback $f^{*} u$. Then $X$ has a divisor $N$ which is contained in the fibers of $f$ but does not contain any fiber such that: 
i) $\pi_{1}(X \backslash N)$ is infinite.

ii) If $f^{*} u$ vanishes on any fiber, then $\pi_{1}(X \backslash N)$ is hyperbolic.

Proof. One can assume the fibers of the map $f$ are connected (if $f$ is not connected use the connected map from the Stein factorization of $f$ and the fact that the orbifold symmetric differentials are preserved via pullback by the finite map in the factorization). If the genus of $C$ is $g \geq 1$, then i) is immediate and ii) follows from the arguments described below for the case $C=\mathbb{P}^{1}$ plus the fact that the orbifold fundamental group of an elliptic curve with nontrivial orbifold structure is hyperbolic.

What remains is the case $C=\mathbb{P}^{1}$. An orbifold symmetric differential $u$ of $\mathbb{P}^{1}$ of degree $m$ is a meromorphic section of $K_{\mathbb{P}^{1}}^{\otimes m}$ whose poles, $\left.u\right|_{U_{x}}=z^{-l} d z^{m}$, have order $l<m$ (for $z^{-l} d z^{m}$ to be resolved via a covering map $t \rightarrow t^{d}$ it needs $l<m)$. Since the $\operatorname{deg} K_{\mathbb{P 1}}^{\otimes m}=-2 m$ and the poles of $u$ are of order $<m$, it follows, in particular, that there are at least 3 distinct poles.

More generally, $u$ has $r$ poles $p_{i} \in \mathbb{P}^{1}$ with orders $l_{i}, i=1, \ldots, r$ for which

$$
\sum_{i=1}^{r} l_{i}=2 m
$$

The condition that the pullback $f^{*} u$ is holomorphic implies that the multiplicities of irreducible components $D_{i j}$ of the fibers $F_{i}=f^{-1}\left(p_{i}\right)=\sum_{j} d_{i j} D_{i j}$ satisfy:

$$
d_{i j} \geq m /\left(m-l_{i}\right)
$$

This follows from the fact that the pullback of $z^{-l}(d z)^{m}$ via a map $\psi:(\Delta, t) \rightarrow$ $(\Delta, z)$ given by $\psi(t)=t^{d}$ is $t^{(d-1) m-d l}(d t)^{m}$. Let $d_{i}=\min \left(d_{i j}\right)$, then (3.7) gives also $d_{i} \geq m /\left(m-l_{i}\right)$.

Denote by $D_{i} \subset F_{i}$ the divisor that is union of all the $D_{i j}$ which have multiplicity $d_{i}, N_{i}$ the union of all irreducible components of $F_{i}$ not in $D_{i}$ and $N=\cup N_{i}$. The fibration $f: X \rightarrow \mathbb{P}^{1}$ implies the following surjection between the fundamental group of $X \backslash N$ and the orbifold fundamental group of $\mathbb{P}^{1}$ with orbifold structure given by the multiple fibers $D_{i}$ (see for example [CaKeOg]).

$$
\pi_{1}(X \backslash N) \rightarrow \pi_{1}^{\text {orb }}\left(\mathbb{P}^{1} \mid\left(d_{1}, \ldots, d_{r}\right)\right) \rightarrow 1
$$

$\pi_{1}^{\text {orb }}\left(\mathbb{P}^{1} \mid\left(d_{1}, \ldots, d_{r}\right)\right)=\pi_{1}\left(\mathbb{P}^{1} \backslash\left\{p_{1}, \ldots, p_{r}\right\}\right) / R_{d_{1} \ldots d_{r}}, R_{d_{1} \ldots d_{r}}$ is the smallest normal subgroup of $\pi_{1}\left(\mathbb{P}^{1} \backslash\left\{p_{1}, \ldots, p_{r}\right\}\right)$ containing the $\gamma_{i}^{d_{i}}$ where the $\gamma_{i}$ are the simple loops around $p_{i}$ 's. 
The orbifold fundamental group $\pi_{1}^{\text {orb }}\left(\mathbb{P}^{1} \mid\left(d_{1}, \ldots, d_{r}\right)\right)$ is infinite if

$$
\sum_{i=1}^{r} 1 / d_{i} \leq r-2
$$

and is hyperbolic if the strict inequality holds. Finally the bounds (3.7) and the equality (3.6) give exactly (3.9) $\left(\sum_{i=1}^{r} 1 / d_{i} \leq \sum_{i=1}^{r}\left(m-l_{i}\right) / m=r-2\right)$. Hence item i) is proved.

The hypothesis of item ii) $\left(f^{*} u\right.$ vanishes along a fiber of $\left.f\right)$, implies that one of following holds: a) $u$ vanishes somewhere on $\mathbb{P}^{1}$; b) $f^{*} u$ vanishes along one of the fibers $F_{i}$ above the poles of $u$; c) $f^{*} u$ vanishes along a fiber $f^{-1}(p)$ not over a pole. The case a) and b) imply respectively that $\sum_{i=1}^{r} l_{i}>2 m$ and one of the $d_{i}>m /\left(m-l_{i}\right)$, hence $\sum_{i=1}^{r} 1 / d_{i}<r-2$ and the hyperbolicity of $\pi_{1}(X \backslash N)$ follows. The case c) implies that the fiber $f^{-1}(p)$ is also multiple and hence the surjection $\pi_{1}(X \backslash N) \rightarrow \pi_{1}^{\text {orb }}\left(\mathbb{P}^{1} \mid\left(d_{1}, \ldots, d_{r}, d_{p}\right)\right)$ with $d_{p}$ the multiplicity of the fiber over $p$ holds. Since $\sum_{i=1}^{r} 1 / d_{i}+1 / d_{p}<(r+1)-2$ the hyperbolicity of $\pi_{1}(X \backslash N)$ is again guaranteed.

Corollary 3.6. Let $X$ be a projective manifold with a symmetric differential $w \in H^{0}\left(X, S^{m} \Omega_{X}^{1}\right)$ of rank 1 induced from a curve (via the map $f: X \rightarrow C$ ). Then either:

i) $\pi_{1}(X)$ is infinite.

or

ii) $\exists N$ a divisor with $\pi_{1}(X \backslash N)$ hyperbolic and whose connected components $N_{i} \subsetneq F_{i}$, where $F_{i}$ are fibers of $f$.

Proof. If the divisor of zeros $(w)_{0}$ is trivial, then by theorem $2.3 \pi_{1}(X)$ is infinite. Otherwise by hypothesis and theorem 3.2 there is a fibration $f: X \rightarrow C$ onto a curve $C$ with $f^{*} u=w, u$ an orbifold symmetric differential on $C$. The divisor of zeros $(w)_{0}$ must be contained in the fibers of $f$ and we are in the case ii) of theorem 3.5 concluding the proof.

Concerning what negativity properties one can obtain on divisors whose complement has infinite fundamental group, the next definition sets the goal.

Definition 3.7. A divisor $D$ on a projective manifold is said to be s-negative if its intersection $D \cap S$ with a complete intersection $S$ of dimension 2 is a negative divisor of $S$. 
Corollary 3.8. If $X$ is a projective manifold with a nontrivial symmetric differential of rank 1 , then there is a s-negative divisor $N$ with $\pi_{1}(X \backslash N)$ infinite.

Proof. The Structure Theorem proves the result with $N=E$ if $\operatorname{dim} a_{w}(X) \geq 2$. The case when the image $a_{w}(X)$ is a curve is settled using the $N$ of corollary 3.6 .

\subsection{Twisted symmetric differentials of rank 1 .}

In this section one considers rank 1 twisted symmetric differentials, $w \in$ $H^{0}\left(X, S^{m} \Omega_{X}^{1} \otimes L\right)$, where $L$ is a $\mathbb{C}^{*}$-flat line bundle on $X$ (or equivalently $L$ has a trivial complex Chern class). The space of $\mathbb{C}^{*}$-flat line bundle on $X$ will be denoted by $P i c^{\tau}(X)$. Twisted symmetric differentials of rank 1 inherit some of the geometric properties of twisted holomorphic differentials which were studied in [GrLa], $[\mathrm{Be}],[\mathrm{Si}]$ and $[\mathrm{Ar}]$ to understand the cohomology locus $S^{1}(X)=\left\{L \in \operatorname{Pic}^{\tau}(X) \mid H^{1}(X, L) \neq 0\right\}$. The relevance of twisted symmetric differentials of rank one on the topic of closed symmetric differentials comes from the fact that they appear naturally in the decomposition of closed symmetric differentials of higher rank, see [BoDeO10].

Theorem 3.9. Let $X$ be a projective manifold with a nontrivial twisted symmetric differential of rank $1, w \in H^{0}\left(X, S^{m} \Omega_{X}^{1} \otimes L\right)$, for a non-torsion $L \in \operatorname{Pic}^{\tau}(X)$. Then $\exists l \in \mathbb{N}^{+}$for which there is a holomorphic map into a smooth curve of genus $\geq 1, p: X \rightarrow B$, such that:

i) $L^{\otimes l}=p^{*} L_{0}$ with $L_{0} \in \operatorname{Pic}^{0}(B)$

ii) $w^{\otimes l}=p^{*} u$ with $u \in H^{0}\left(B, S_{\text {orb }}^{l m} \Omega_{B}^{1} \otimes L_{0}^{\otimes l}\right)$.

Proof. The line bundle $L \in \operatorname{Pic}^{\tau}(X)$ might not be divisible by $m$. So one does the abelian Galois covering $h: Y \rightarrow X$ associated with the surjection of $\pi_{1}(X)$ to $H_{1}(X, \mathbb{Z})_{\text {tor }}$ to make $h^{*} L \in P i c^{0}(Y)$ and hence divisible. Let $L_{1}$ be such that $L_{1}^{\otimes m}=h^{*} L$, then $h^{*} w \in H^{0}\left(Y, S^{m}\left(\Omega_{Y}^{1} \otimes L_{1}\right)\right)$, before proceeding set $w_{1}=h^{*} w$. The next step is the use of the straightforward generalization of theorem 2.3 for $\Omega_{Y}^{1} \otimes L_{1}$ that gives a finite cyclic ramified covering $f: Y^{\prime} \rightarrow Y$ such that $f^{*} w_{1}=\mu^{\otimes m}$ with $\mu \in H^{0}\left(Y^{\prime}, \Omega_{Y^{\prime}}^{1} \otimes f^{*} L_{1}\right)$.

One then proceeds to apply the results of [Gr-La87], [Be92], [Si93]. Set $L_{2}=$ $f^{*} L_{1}, L_{2}$ being unitary gives $H^{1}\left(Y^{\prime}, L_{2}^{*}\right) \simeq H^{0}\left(Y^{\prime}, \Omega_{Y^{\prime}}^{1} \otimes L_{2}\right)$ and hence its dual 
$L_{2}^{*} \in S^{1}\left(Y^{\prime}\right)=\left\{L \in \operatorname{Pic}^{\tau}\left(Y^{\prime}\right) \mid H^{1}\left(Y^{\prime}, L\right) \neq 0\right\}$. Since $L_{2}^{*}$ is non torsion, the work of Simpson [Si93] gives that $L_{2}^{*}$ must be in a positive dimensional component of $S^{1}\left(Y^{\prime}\right)$. The results of Green-Lazarsfeld [Gr-La87], then imply that there exists a $v \in H^{0}\left(Y^{\prime}, \Omega_{Y^{\prime}}^{1}\right)$ such that the following sequence, coming from a derivative complex, is totally not exact at the middle:

$$
H^{0}\left(Y^{\prime}, L_{2}\right) \stackrel{\wedge v}{\longrightarrow} H^{0}\left(Y^{\prime}, \Omega_{Y^{\prime}}^{1} \otimes L_{2}\right) \stackrel{\wedge v}{\longrightarrow} H^{0}\left(Y^{\prime}, \Omega_{Y^{\prime}}^{2} \otimes L_{2}\right)
$$

that is, all $\alpha \in H^{0}\left(Y^{\prime}, \Omega_{Y^{\prime}}^{1} \otimes L_{2}\right)$ with $\alpha \wedge v=0$ are such that $\alpha \neq v \otimes \phi$ with $\phi \in H^{0}\left(Y^{\prime}, L_{2}\right)$.

The above gives in particular that there is a $v \in H^{0}\left(Y^{\prime}, \Omega_{Y^{\prime}}^{1}\right)$ such that $\mu \wedge$ $v=0$ and $\mu \neq v \otimes \phi$ with $\phi \in H^{0}\left(Y^{\prime}, L_{2}\right)$ (a non-proportionality condition). These two conditions give due to Beauville [Be92] a Castelnouvo-De Franchis type theorem. The Castelnuovo-De Franchis type theorem states that $Y^{\prime}$ has a connected holomorphic map, $q^{\prime}: Y^{\prime} \rightarrow C^{\prime}$, onto a curve of genus $g \geq 1$,

$$
\mu \in H^{0}\left(Y^{\prime}, q^{\prime *} \Omega_{C^{\prime}}^{1} \otimes L_{2}\right)
$$

and $L_{2} \in P i c^{\tau}\left(X, q^{\prime}\right)$ (i.e $L_{2}$ is flat and is trivial in one fiber of $q^{\prime}$ ).

The cyclic action on $Y^{\prime}$ and the abelian action on $Y$ have respectively $f^{*} w_{1}$ and $w_{1}=h^{*} w$ as an invariant. Hence from the connected map $q^{\prime}: Y^{\prime} \rightarrow C^{\prime}$, whose fibers are the leaves of the foliation defined by $f^{*} w_{1}$, one obtains the following commutative diagram where the horizontal maps are connected and their targets smooth curves.

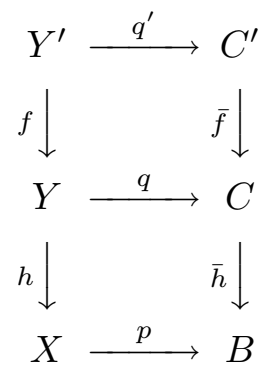

The map $\bar{f}$ is a cyclic ramified covering and $\bar{h}$ is an unramified Galois covering.

The line bundle $L \in P i c^{\tau}(X, p)$ since $L_{2} \in \operatorname{Pic}^{\tau}\left(Y^{\prime}, q^{\prime}\right)$. This implies $L=$ $p^{*} H \otimes \mathcal{O}\left(\sum k_{i} F_{i}\right)$ with $H \in P i c^{0}(B), F_{i}$ the multiple fibers of $p$ and $\sum k_{i} / m_{i}=0$ ( $m_{i}$ the multiplicities of the multiple fibers). If $l=$ l.c. $m\left\{m_{i}\right\}$, then line bundle $\mathcal{O}\left(\sum k_{i} F_{i}\right)^{\otimes l} \in p^{*} P i c^{0}(B)$. Hence there is a $L_{0} \in P i c^{0}(B)$ such that 


$$
L^{\otimes l}=p^{*} L_{0}
$$

and hence $g(B) \geq 1$ since $L_{0}$ must be non-torsion. From (3.11), (3.12) and (3.13) it follows that $\mu^{\otimes m l}=q^{\prime *} t^{\prime}$ with $t^{\prime} \in H^{0}\left(C^{\prime}, S^{m l} \Omega_{C^{\prime}}^{1} \otimes(\bar{h} \circ \bar{f})^{*} L_{0}\right)$. The twisted symmetric differential $t^{\prime}$ is invariant under the cyclic action on $C^{\prime}$ and gives and element $t \in H^{0}\left(C, S_{\text {orb }}^{m l} \Omega_{C}^{1} \otimes \bar{h}^{*} L_{0}\right)$ which is invariant for the abelian action on $C$ and hence gives an the desired $u \in H^{0}\left(B, S_{o r b}^{l m} \Omega_{B}^{1} \otimes L_{0}^{\otimes l}\right)$.

Corollary 3.10. A twisted symmetric differential of rank $1, w \in H^{0}\left(X, S^{m} \Omega_{X}^{1} \otimes\right.$ $L)$, with $L \in \operatorname{Pic}^{\tau}(X)$ non-torsion defines a singular holomorphic foliation $\mathcal{F}_{w}$ on $X$ which is a fibration over a curve.

Proof. Immediate consequence of the previous theorem.

Theorem 3.11. Let $X$ be a projective manifold with $w \in H^{0}\left(X, S^{m} \Omega_{X}^{1} \otimes L\right)$ a nontrivial twisted symmetric differential of rank 1. Then:

i) if $L$ is torsion, then there is a s-negative divisor $N$ of $X$ such that $\pi_{1}(X \backslash N)$ has a subgroup $G$ of finite index with infinite abelianization, $G /[G, G]$.

ii) if $L$ is non torsion, then $\pi_{1}(X)$ surjects onto $\pi_{1}(B)$ with $B$ a curve with $g(B) \geq 1$ and there is a s-negative divisor $N$ of $X$ such that $\pi_{1}(X \backslash N)$ is hyperbolic.

Proof. i) follows from corollary 3.8 since there is an $l$ such that $L^{\otimes l}=\mathcal{O}$ and hence $w^{\otimes l}$ is an ordinary symmetric differential of rank 1 on $X$.

ii) is a consequence of theorem 3.9 and the arguments of theorem 3.5. Consider the holomorphic map $p: X \rightarrow C$ built in theorem 3.9 where $g(C) \geq 1$. If none of the fibers of $p$ has multiplicity $\geq 2$, then the twisted symmetric differential $u=H^{0}\left(B, S_{o r b}^{l m} \Omega_{B}^{1} \otimes L_{0}^{\otimes l}\right)$ such that $w^{\otimes l}=p^{*} u$ found in theorem 3.9 would be in $H^{0}\left(C, S^{l m} \Omega_{C}^{1} \otimes L_{0}^{\otimes l}\right)$. This situation would force $g(C) \geq 2$ since $L_{0}$ is nontorsion and $\Omega_{C}^{1} \simeq \mathcal{O}$ if $C$ is elliptic. If some fibers have multiplicity $\geq 2$ then either $\pi_{1}(X)$ is hyperbolic (case all the irreducible components of the multiple fibers have multiplicity equal to the multiplicity of the respective fiber) or set $N$ to be the union of all the irreducible components of the fibers whose multiplicity differs from the multiplicity of the respective fiber. The conclusion follows from the same arguments used in theorem 3.5. 
SYMMETRIC DIFFERENTIALS OF RANK 1 AND HOLOMORPHIC MAPS 1103

\section{REFERENCES}

[AbWa97] D.Abramovich, J.Wang, Equivariant resolution of singularities in characteristic O, Math. Res. Letters 4 (2-3) (1997), 427-433.

[Be92] A.Beauville, Annulation du $H^{1}$ pous les fibres en droites plats, Complex Algebraic Varieties, Proc. Conf., Bayreuth, 1990, Springer lecture Notes in Math 1507 (1992), 1-15.

[BoDeO06] F.Bogomolov, B.De Oliveira, Hyperbolicity of nodal hypersurfaces, J. Regne Angew. Math. 596 (2006).

[BoDeO10] F.Bogomolov, B.De Oliveira, The geometry of closed symmetric 2-differentials on surfaces, Preprint (2010).

[Bo78] F.Bogomolov, Holomorphic tensors and vector bundles, Izvestya A.N. USSR 42 (6) (1978), 1227-1287 (English translation Math USSR Izvestya 1979 v 13 (1) 499-544).

[Gr-La87] M.Green, R.Lazarsfeld, Deformation theory, generic vanishing theorems, and some conjectures of Enriques, Catanese and Beauville, Invent. Math. 90 (1987), 389-407.

[La04] R. Lazarsfeld, Positivity in algebraic geometry, Springer Verlag, Ergebnisse series vol. 48, 49, 2004.

[Si93] C.Simpson, Subspaces of moduli spaces of rank one local systems, Ann. scient. Ec. Norm. Sup., $4^{e}$ serie 26 (1993), 361-401.

Fedor Bogomolov

Courant Institute for Mathematical Sciences,

New York University and Laboratory of Algebraic Geometry,

GU-HSE, 7 Vavilova Str., Moscow, Russia, 117312

E-mail: bogomolo@CIMS.NYU.EDU

Bruno De Oliveira

University of Miami

E-mail: bdeolive@math.miami.edu 